\title{
Mise en bouche
}

\section{Bernhard Gurtner}

Dr méd., ancien médecin-chef de I'hôpital de Wetzikon, membre de la SSMIG

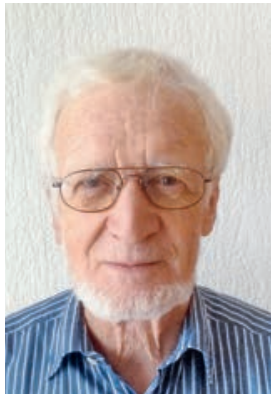

Un étudiant en médecine se promenait à Mexico avec son chien, qui reniflait avec curiosité tout ce qu'il trouvait sur son chemin. L'animal s'écroula soudain sur le sol en râlant. Létudiant, formé aux premiers secours, entreprit immédiatement un bouche-à-gueule, mais fut lui-même intoxiqué, vomit et perdit conscience. Le chien est mort. L'étudiant a pu être sauvé par l'hôpital le plus proche, grâce au médecin urgentiste qui avait identifié une odeur d'amande amère, caractéristique de l'acide cyanhydrique, et avait immédiatement appliqué un traitement efficace.

Le bouche-à-groin réalisé dans un zoo de Hanovre a mieux réussi à l'animal et a permis au soigneur de sauver la vie d'un bébé tapir. Depuis toujours, les éleveurs de bétail et les vétérinaires sauvent les nouveau-nés souffrant d'asphyxie grâce à la respiration artificielle. Désormais, un ballon de ventilation leur évite d'avoir à insuffler l'air aux petits veaux ou aux porcelets à partir de leur propre bouche.

L'étudiant, formé aux premiers secours, entreprit immédiatement un bouche-à-gueule.

Dans les piscines, on trouvait autrefois des instructions affichées, expliquant les gestes de réanimation de la méthode Holger-Nielsen à appliquer sur un noyé. Cette technique étant rarement efficace, elle a été remplacée à partir de 1960 par un procédé de respiration artificielle proche du bouche-à-bouche, puis peu de temps après par le bouche-à-nez, qui génère en effet moins de répugnance et de craintes au niveau de l'hygiène. Sans compter que les nez proéminents offrent aux souffleurs un meilleur point d'attaque que les lèvres fines.

Les bouches en contact ne sont pas seulement le thème favori des films et romans torrides. C'est aussi un danger pour la santé, sur lequel les amoureux préfèrent généralement fermer les yeux. L'humanité a survécu malgré (ou grâce à) cela. La mononucléose, très répandue chez les adolescents, est aussi appelée «kissing disease», car elle se transmet en s'embrassant. Elle rend les médecins-assistants perplexes quand elle touche deux laborantines hospitalières en même temps.
Mais les agents pathogènes infectieux ne sont pas les seuls à être transmis par un simple baiser. Une jeune femme est tombée en état de choc anaphylactique après avoir embrassé son petit ami qui venait de manger une crevette. La patiente avait déjà réagi au homard et aux crevettes par de violentes crises d'urticaire,

\section{Dans les EMS, les prothèses ont parfois été} mélangées au moment du nettoyage.

mais ne se doutait pas qu'un simple baiser pour se souhaiter bonne nuit pouvait avoir des conséquences aussi renversantes. Les patients allergiques aux cacahouètes sont eux aussi sensibles à la moindre trace, et doivent demander à leur chéri(e) de bien se rincer la bouche s'il/elle a mangé des fruits à coque. Cela ne suffit pas toujours et, parfois, il faut choisir entre bisou et noix de cajou.

Dans les EMS, les prothèses ont parfois été mélangées au moment du nettoyage, ce qui en a amené certains à se disputer et à montrer les dents. Une élève infirmière a eu toutes les peines du monde à retrouver les propriétaires de prothèses dentaires qu'elle avait nettoyées dans le même lavabo. On fait parfois du zèle pour rationaliser dans les milieux de la santé.

Il est même arrivé qu'un couple démuni soit obligé de se rationner et de manger en décalé, parce que les époux ne pouvaient se payer qu'une prothèse pour deux. Nos grands-parents appareillés avaient coutume de raconter cette anecdote peu appétissante. J’ai moimême rencontré des descendants se querellant à l'hôpital pour récupérer la prothèse dentaire laissée par leur proche qui venait de mourir paisiblement. On était tellement économe dans notre pays, que les jeunes qui avaient des caries recevaient une prothèse dentaire dès leur confirmation. Mais peut-être que cela n'est qu'une rumeur, racontée de bouche en bouche, jusqu'à ce que les hygiénistes dentaires nous proposent une nouvelle génération de dents impeccablement blanches, fièrement arborées via les sourires des selfies sur les réseaux sociaux. 